\title{
A Generalization of Bochner's Tube Theorem in Elliptic Boundary Value Problems
}

\author{
Dedicated to Professor H. Komatsu on his sixtieth birthday
}

By

\author{
Motoo UcHIDA*
}

\section{Contents}

Introduction

Notations

§1. Main Theorem

§2. Elliptic boundary value problems

§3. Proof of Theorem 1.1

§4. A tempered version of Theorem 1.1

Appendix A. A diagram in Fourier-Sato transformation of sheaves

Appendix B. Boundary value morphism and microlocalization

\section{Introduction}

It is classically well known as Bochner's tube theorem that any holomorphic function defined on a tube domain $T$ in a complex affine space has analytic continuation on the convex full of $T$ [H, Theorem 2.5.10].

Now let $M$ be a real analytic manifold, $X$ its complexification. Let $T_{M} X$ be the normal bundle of $M$ in $X, \nu_{M}$ the functor of specialization along $M$, and let $\widetilde{\mathscr{A}}_{M}$ denote the sheaf $H^{0} \nu_{M}\left(\mathcal{O}_{X}\right)$ on $T_{M} X$. In [SKK, Chap. I], in connection with the theory of microfunctions, a proof is given to the following local version of Bochner's tube theorem :

Theorem. Let $U$ be an open conic subset of $T_{M} X$ with connected fibres, $\widetilde{U}$ the convex full of $U$ in each fibre. Then

Communicated by M. Kashiwara, April 14, 1995.

1991 Mathematics Subject Classification: 58G05, 35A27.

* Department of Mathematics, Osaka University, Machikaneyama 1-16, Toyonaka, Osaka 560 , Japan. 


$$
\Gamma\left(\widetilde{U}, \tilde{\mathscr{A}}_{M}\right) \rightarrow \Gamma\left(U, \widetilde{\mathscr{A}}_{M}\right)
$$

is an isomorphism.

We can refer to $[\mathrm{H}$, Lemma 2.5.10] and [Ko] for a more quantitative local version of Bochner's theorem and its application.

This kind of microlocal analytic continuation theorem is also proved for a generic real analytic submanifold $M$ of a complex manifold $X$ (see Example 1.2). This remark is made by Aoki and Tajima [AT2] and by Baouendi and Rothschild [BR].

In this article, considering a real setting, we prove a similar local version of Bochner's theorem in boundary value problems for elliptic systems of differential equations on a real manifold $X$. If $X$ is a complex manifold, we can recover the result of [AT2]. For the details, see Section 1.

The proof, presented in Sections 2 and 3, is an analogue of that of [SKK, Chap. I, Prop. 1.5.4]. The key fact is a cohomology vanishing theorem due to Kashiwara and Kawai $[\mathrm{KK}]$ for solution complexes of elliptic coherent $\mathscr{D}$-modules (see Section 2). As in [SKK, Sect. 1.5], the technical basis of the arguments is the use of the Fourier-Sato transformation of sheaves. For every morphism $f: X \rightarrow Y$ of locally compact spaces, with $f_{1}$ of finite cohomological dimension, there is a canonical morphism id $\rightarrow f^{!} \mathbf{R} f_{1}$ of functors, which we call the adjoint trace morphism for sheaves. In appendix $\mathrm{A}$, we give a triangle in the derived category which describes a relation of the adjoint trace morphism of sheaves on a vector bundle to their Fourier transforms (see Corollary A.2). We effectively use this triangle in the proof of Main Theorem. (The same triangle on sphere bundles is found also in [SKK, Chap.I, Proposition 1.4.4], where it is deduced by the octahedral axiom of the triangulated category. In Appendix A, without using the octahedral axiom, we establish a more basic commutative diagram, which yields the required triangle as an immediate corollary.)

In Section 4, using a result [AT1] on elliptic boundary value problems in the space of tempered distributions, we state a proposition of the same type as Main Theorem on microlocal analytic continuation with a growth condition.

In Appendix A, we review a formalism of the Fourier-Sato transformation of sheaves [KS, Sect. 3.7] and prove a few canonical commutative diagrams coming from the adjoint trace morphism $G \rightarrow \tau^{!} \mathbf{R} \tau_{!} G$ for sheaves on vector bundles. This section is the main geometric part of this paper.

In Appendix B, we summarize the construction of boundary value morphisms as adjoint trace morphism for normal bundles, which construction appears naturally in boundary value problems, especially of higher codimension, as we see in Section 3. We also discuss its relation to microlocalization. 


\section{Notations}

In these notes we freely use the notations of [KS].

We always assume a manifold to be paracompact. For a field $k$ of characteristic zero or positive, we denote by $\mathrm{D}\left(k_{X}\right)$ the derived category of the category of complexes of $k_{X}$-modules on a topological manifold $X$, by $\mathrm{D}^{\mathrm{b}}\left(k_{X}\right)$ or simply by $\mathrm{D}^{\mathrm{b}}(X)$ the full subcategory of $\mathrm{D}\left(k_{X}\right)$ consisting of $F \in \mathrm{Ob}\left(\mathrm{D}\left(k_{X}\right)\right)$ such that $H^{j}(F)=0$ if $|j| \gg 0$. Except in appendices, with $k$ being the complex number field, $\mathrm{D}^{\mathrm{b}}(X)$ signifies $\mathrm{D}^{\mathrm{b}}\left(\mathbf{C}_{X}\right)$.

If $E$ is a vector bundle over a topological manifold, $D_{\mathbf{R}^{+}}^{b}(E)$ denotes the full subcategory of $\mathrm{D}^{\mathrm{b}}(E)$ such that

$$
\begin{aligned}
\mathrm{Ob}\left(\mathrm{D}_{\mathbf{R}^{+}}^{\mathrm{b}}(E)\right)=\left\{F \in \mathrm{Ob}\left(\mathrm{D}^{\mathrm{b}}(E)\right) \mid H^{j}(F)\right. & \text { is locally constant } \\
& \text { on each } \left.\mathbf{R}^{+} \text {-orbit of } E, \forall j \in \mathbf{Z}\right\} .
\end{aligned}
$$

For a complex manifold $Z, O_{Z}$ denotes the sheaf of holomorphic functions on $Z$ and $\mathscr{D}_{Z}$ the sheaf of differential operators with holomorphic coefficients on $Z$.

\section{§ 1. Main Theorem}

Let $X$ be a real analytic manifold, $\mathscr{A}_{X}$ the sheaf of analytic functions on $X$. Let $M$ be a closed submanifold of $X$ of codimension $d \geq 1$. We denote by $\mathscr{D}_{X}$ the sheaf of rings of differential operators with analytic coefficients on $X$.

Let $\mathscr{M}$ be a coherent left $\mathscr{D}_{X}$-module defined on $X$. We assume the following conditions :

(a.1) $\mathscr{M}$ is elliptic :

$$
T_{X}^{*} W \cap \operatorname{Ch}(\mathscr{M}) \subset T_{W}^{*} W
$$

where $W$ is a complex neighborhood of $X$ on which $\mathscr{M}$ has analytic continuation as coherent $\mathscr{D}_{W}$-module, and $\mathrm{Ch}(\mathscr{M})$ denotes the characteristic variety of $\mathscr{M}$.

(a.2) The complexification $Z$ of $M$ in $W$ is non characteristic for $\mathscr{M}$ :

$$
T_{Z}^{*} W \cap \operatorname{Ch}(\mathscr{M}) \subset T_{W}^{*} W .
$$

We set: $\mathscr{A}_{X}^{\bullet}=\mathbf{R} \mathscr{H} o m_{\mathscr{D}_{X}}\left(\mathscr{M}, \mathscr{A}_{X}\right)$. If $\mathscr{M}$ has a free resolution

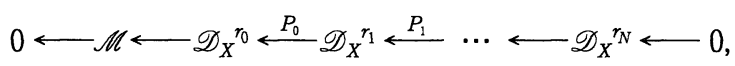

with $P_{k}$ being an $r_{k+1} \times r_{k}$ matrix of differential operators acting on the right, $\mathscr{A}_{X}{ }^{\bullet}$ is represented by 


$$
0 \longrightarrow \mathscr{A}_{X}^{r_{0}} \stackrel{P_{0}}{\longrightarrow} \mathscr{A}_{X}^{r_{1}} \stackrel{P_{1}}{\longrightarrow} \cdots \longrightarrow \mathscr{A}_{X}^{r_{N}} \longrightarrow 0
$$

Let $\tau: T_{M} X \rightarrow M$ be the normal bundle of $M$ in $X$. Recalling the specialization functor [KS, Sect. 4.2]

$$
\nu_{M}: \mathrm{D}^{\mathrm{b}}(X) \rightarrow \mathrm{D}_{\mathrm{R}^{+}}^{\mathrm{b}}\left(T_{M} X\right),
$$

we have :

Theorem 1.1. Let $U$ be an open conic subset of $T_{M} X$ with connected fibres, $\widetilde{U}$ the convex hull of $U$ in each fibre. Then

$$
\Gamma\left(\widetilde{U}, H^{0} \nu_{M}\left(\mathscr{A}_{X}^{\bullet}\right)\right) \rightarrow \Gamma\left(U, H^{0} \nu_{M}\left(\mathscr{A}_{X}^{\bullet}\right)\right)
$$

is an isomorphism.

Example 1.2. Let $\left(X_{\mathrm{C}}, \mathcal{O}_{X_{\mathrm{C}}}\right)$ be a complex manifold, $X$ the underlying real manifold of $X_{\mathrm{C}}, M$ a generic real analy tic submanifold of $X_{\mathrm{C}}$. (A submanifold $M$ of $X_{\mathrm{C}}$ is said to be generic if $T M+i T M=T X \times_{X} M$.) Let $\mathscr{M}_{\mathrm{CR}}$ be the Cauchy-Riemann system on $X: \mathscr{M}_{\mathrm{CR}}=\delta^{-1}\left(q_{2}{ }^{*}{O_{\bar{X}}}_{\overline{\mathrm{C}}_{\mathrm{C}}}\right)$, where $\bar{X}_{\mathrm{C}}$ is the conjugate complex manifold of $X_{\mathrm{C}}, q_{2}: X_{\mathrm{C}} \times \bar{X}_{\mathrm{C}} \rightarrow \bar{X}_{\mathrm{C}}$ the projection, $\delta: X \hookrightarrow X \times \bar{X}$ the diagonal embedding, and $q_{2}{ }^{*}$ denotes the inverse image functor of $\mathscr{D}$-modules (cf. for example [T]). Then $\left(X, M, \mathscr{M}_{\mathrm{CR}}\right.$ ) satisfies conditions (a.1) and (a.2), and

$$
\mathbf{R} \mathscr{H O m _ { \mathscr { D } _ { X } }}\left(\mathscr{M}_{\mathrm{CR}}, \mathscr{A}_{X}\right) \cong \mathcal{O}_{X_{\mathrm{C}}}
$$

Hence the theorem above holds for the sheaf $H^{0} \nu_{M}\left(\mathcal{O}_{X_{\mathrm{C}}}\right)$; this is a microlocal version of Bochner's tube theorem for a generic CR-submanifold $M$, proved by Aoki and Tajima [AT2] and by Baouendi and Rothschild [BR, Sect. 3] (cf. also [BRT, part 2]).

If $M$ is a real analytic manifold embedded in its complexification $X_{\mathrm{C}}$, this example makes us regain the classical local version of Bochner's tube theorem [SKK, Chap. I, Prop. 1.5.4].

\section{§ 2. Elliptic Boundary Value Problems}

Let $M, X, \mathscr{M}$ be as in Section 1: $\mathscr{M}$ is an elliptic system of differential equations on $X$, and the complexification of $M \rightarrow X$ is non characteristic for $\mathscr{M}$.

Let $\pi: T_{M}^{*} X \rightarrow M$ be the conormal bundle of $M$ in $X$. Recalling the Sato microlocalization functor (cf. $[\mathrm{KK}]$ and $[\mathrm{KS}$, Sect. 4.3$]$ ) 


$$
\mu_{M}: \mathrm{D}^{\mathrm{b}}(X) \rightarrow \mathrm{D}_{\mathrm{R}^{+}}^{\mathrm{b}}\left(T_{M}^{*} X\right)
$$

we have :

Theorem 2.0. For $j<d, H^{\jmath} \mu_{M}\left(\mathscr{A}_{X}^{\bullet}\right)=0$.

This is a conclusion of the isomorphism of Kashiwara and Kawai [KK].

\section{§ 3. Proof of Theorem 1.1}

Let $M, X, \mathscr{M}$ be as in Section 1 , and set $G=\nu_{M}\left(\mathscr{A}_{X}^{\bullet}\right)$; then $G$ is an object of $\mathrm{D}_{\mathbf{R}^{+}}^{\mathrm{b}}\left(T_{M} X\right)$ and by definition $G^{\wedge}=\mu_{M}\left(\mathscr{A}_{X}^{\bullet}\right)$. By Theorem 2.0 , we have $H^{j} G^{\wedge}=0$ for $j<d$. ( $G^{\wedge}$ denotes the Fourier-Sato transform of $G$; for the notations in Fourier-Sato transformations, see Appendix A.)

Hence, from Corollary A.2, we have an exact sequence of sheaves on $T_{M} X$ :

$$
0 \rightarrow H^{0} G \rightarrow \tau^{-1} H^{d} \mathbf{R} \tau_{!} G \otimes o r_{M \mid X} \rightarrow p_{1 *}^{+} p_{2}^{+-1}\left(H^{d} G^{\wedge} \otimes \pi^{-1} o r_{M \mid X}\right)
$$

where $o r_{M \mid X}$ denotes the relative orientation sheaf of $M$ in $X$. Let $U$ be an open conic subset of $T_{M} X, U^{\supset a}$ its antipodal polar set in $T_{M}^{*} X$. From (3.1), we have

$$
\begin{aligned}
0 \rightarrow \Gamma\left(U, H^{0} G\right) \rightarrow \Gamma\left(M, H^{d} \mathbf{R} \tau_{1}\right. & \left.G \otimes o r_{M \mid X}\right) \\
& \rightarrow \Gamma\left(T_{M}^{*} X \backslash U^{\text {oa }}, H^{d} G^{\wedge} \otimes \pi^{-1} o r_{M \mid X}\right) \quad \text { (exact) }
\end{aligned}
$$

if every fibre of $\left.\tau\right|_{U}: U \rightarrow M$ is connected and non-empty. Since $U^{\text {oa }}=\widetilde{U}^{\text {oa }}$, it follows that

$$
\Gamma\left(\widetilde{U}, H^{0} G\right) \rightarrow \Gamma\left(U, H^{0} G\right)
$$

is an isomorphism.

Remark. Let $F=\mathscr{A}_{X}{ }^{\circ}$. Then the second arrow of (3.1) results in

$$
\left.H^{0} \nu_{M}(F) \rightarrow \tau^{-1} H_{M}^{d}(F)\right|_{M} \otimes o r_{M \mid X}
$$

and is regarded as boundary value map for $F$. See Appendix B.

\section{§4. A Tempered Version of Theorem 1.1}

Let $M, X, \mathscr{M}$ be again as in Section 1 . In particular, $\mathscr{M}$ is an elliptic system of differential equations on $X$. Let $\mathscr{D}_{k_{X}}$ be the sheaf of distributions on $X$. 
Recently Andronikof and Tose [AT1] have proved an analogue of the formula of $[\mathrm{KK}]$ in elliptic boundary value problems for tempered distributions. By their result, we have in particular:

Proposition 4.0 [AT1]. For $j<d$,

$$
H^{j} \mathbf{R} \mathscr{H} O m_{\pi^{-1} \mathscr{D}_{X}}\left(\pi^{-1}\left(\left.\mathscr{M}\right|_{M}\right), T-\mu_{M}\left(\mathscr{D} k_{X}\right)\right)=0
$$

Here $T-\mu_{M}\left(\mathscr{D} k_{X}\right)$ is the tempered microlocalization of $\mathscr{D} k_{X}$ along $M$ due to Andronikof; this is, by definition, the Fourier-Sato transform of the conic $\tau^{-1}\left(\left.\mathscr{D}_{X}\right|_{M}\right)$-submodule $T-\nu_{M}\left(\mathscr{D} k_{X}\right)$ of $H^{0} \nu_{M}\left(\mathscr{D} k_{X}\right)$. For an open conic subset $U$ of $T_{M} X$, we have

$$
\Gamma\left(U, T-\nu_{M}\left(\mathscr{D} b_{X}\right)\right) \cong \underset{V}{\lim } T_{M} \Gamma\left(V, \mathscr{D} k_{X}\right),
$$

where $V$ ranges through the family $\mathscr{V}_{U}$ of all open subsets of $X$ satisfying $C_{M}(X \backslash V) \cap U=\varnothing$, and

$$
T_{M} \Gamma\left(V, \mathscr{D} k_{X}\right)=\left\{f \in \mathscr{D}_{X}(V) \mid \text { For any } u \in U\right. \text {, }
$$

there is an open subset $V^{\prime}$ of $V$ such that $C_{M}\left(X \backslash V^{\prime}\right) \nexists u$ and $\left.f\right|_{V^{\prime}}$ is tempered at every point of $\left.\overline{V^{\prime}}\right\}$.

A distribution $g$ on an open set $W$ is called tempered at a point $p \in \bar{W}$ if there exists a distribution $\tilde{g}$ defined in a neighborhood $B$ of $p$ so that $\tilde{g}=g$ on $B \cap W$.

Since $\mathscr{M}$ is coherent over $\mathscr{D}_{X}$, we have

$$
\left[\mathbf{R} \mathscr{H} O m_{\tau^{-1} \mathscr{D} X}\left(\tau^{-1}\left(\left.\mathscr{M}\right|_{M}\right), T-\nu_{M}\left(\mathscr{D} k_{X}\right)\right)\right]^{\wedge} \cong \mathbf{R} \mathscr{H} O m_{\pi^{-1} \mathscr{D} X}\left(\pi^{-1}\left(\left.\mathscr{M}\right|_{M}\right), T-\mu_{M}\left(\mathscr{D} k_{X}\right)\right)
$$

and

$$
H^{0}\left(U, \mathbf{R} \mathscr{H} O m_{\tau^{-1} \mathscr{D X}}\left(\tau^{-1}\left(\left.\mathscr{M}\right|_{M}\right), T-\nu_{M}\left(\mathscr{D} \iota_{X}\right)\right)\right) \cong \lim _{V} T_{M} \Gamma\left(V, \mathscr{H} o m_{\mathscr{D} X}\left(\mathscr{M}, \mathscr{D} \iota_{X}\right)\right),
$$

where, for $V \in \mathscr{V}_{U}, T_{M} \Gamma\left(V, \mathscr{H} o m_{\mathscr{D}_{X}}\left(\mathscr{M}, \mathscr{D}_{\iota_{X}}\right)\right)$ denotes the set of all sections on $V$ tempered at the edge $M$ and is defined in the same manner as above.

Hence, in virtue of Proposition 4.0, by the same argument as in Section 3 with $G=\mathbf{R} \mathscr{H} o m_{\tau^{-1} \mathscr{D} X}\left(\tau^{-1}\left(\left.\mathscr{M}\right|_{M}\right), T-\nu_{M}\left(\mathscr{D} k_{X}\right)\right)$, we obtain the following tempered version of Theorem 1.1:

Proposition 4.1. Let $U$ and $\widetilde{U}$ be as in Theorem 1.1. Then

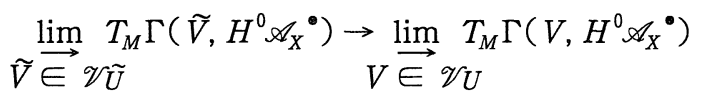


is an isomorphism.

Remark. (4.1) is nothing but morphism (1.1) with a growth condition.

\section{Appendix A. A Diagram in Fourier-Sato Transformation of Sheaves}

In this section, a diagram in Fourier-Sato transformation of conic sheaves on vector bundles will be discussed in the setting of [KS, Sect. 3.7]. We fix a field $k$ of characteristic zero or positive*, and, for a topological manifold $X$, denote by $\mathrm{D}^{\mathrm{b}}(X)$ the derived category $\mathrm{D}^{\mathrm{b}}\left(k_{X}\right)$ of $k_{X}$-modules. Since we work only in the derived category $\mathrm{D}^{\mathrm{b}}\left(k_{X}\right)$, with $k$ a fixed field, we denote simply by $f_{*}, f_{\text {! }}$ the right derived push-forward functors by a continuous map $f$. Except for this simplification, we follow the notations of [KS, loc.cit.] .

For every morphism $f: X \rightarrow Y$ of locally compact spaces, with $f_{\text {! }}$ of finite cohomological dimension, there is a canonical functor $f^{\prime}$ from $\mathrm{D}^{+}\left(k_{Y}\right)$ to $\mathrm{D}^{+}\left(k_{X}\right)$, adjoint to the functor $f_{:}: \mathrm{D}^{+}\left(k_{X}\right) \rightarrow \mathrm{D}^{+}\left(k_{Y}\right)$. There is a canonical morphism id $\rightarrow f^{!} f$ of functors, which we call the adjoint trace morphism for sheaves.

Let $M$ be a $C^{1}$-manifold, $\tau: E \rightarrow M$ a $C^{1}$ vector bundle on $M, \pi: E^{*} \rightarrow M$ the dual bundle of $E$. Consider the diagram

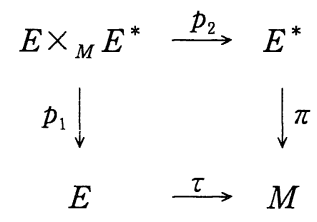

and set:

$$
P^{\prime}=\left\{(x, y) \in E \times{ }_{M} E^{*} \mid\langle x, y\rangle \leq 0\right\}
$$

The Fourier-Sato transformation of sheaves [KS, Sect. 3.7; $c f$. also BMV] is the functor

$$
\Phi: \mathrm{D}_{\mathrm{R}^{+}}^{\mathrm{b}}(E) \rightarrow \mathrm{D}_{\mathbf{R}^{+}}^{\mathrm{b}}\left(E^{*}\right), \quad \Phi(G)=p_{2 !}\left(p_{1}^{-1} G\right)_{P^{\prime}}
$$

for $G \in \mathrm{Ob}\left(\mathrm{D}_{\mathbf{R}^{+}}^{\mathrm{b}}(E)\right)$. Denoting $G^{\wedge}=\Phi(G)$, we have an inversion formula:

Theorem A.0 [KS, BMV]. There is a canonical isomorphism:

$$
G \stackrel{\sim}{\longrightarrow} p_{1 *} \mathbf{R} \Gamma_{P^{\prime}}\left(p_{2}^{!} G^{\wedge}\right) .
$$

* The author thanks the referee for pointing out that the argument works also in the case of characteristic positive. 
The objective of this section is to prove the following commutative diagram.

Lemma A.1. There is a canonical commutative diagram:

(A.1)

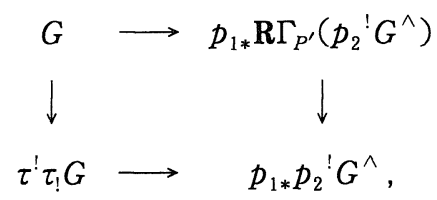

where the left vertical arrow is an adjoint trace morphism and the right one a support morphism $\mathbf{R} \Gamma_{P^{\prime}} \rightarrow \mathbf{R} \Gamma$. In this diagram, every horizontal arrow is an isomorphism.

Proof. The proof is performed by pursuing commutative diagrams. To start with, we have a commutative diagram

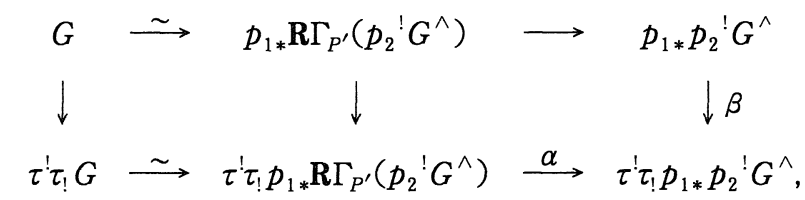

where each horizontal arrow of the left square is an isomorphism in virtue of Theorem A.0. In order to prove both $\alpha$ and $\beta$ in (A.2) to be isomorphisms, we use the following cartesian diagram of $C^{1}$-maps:

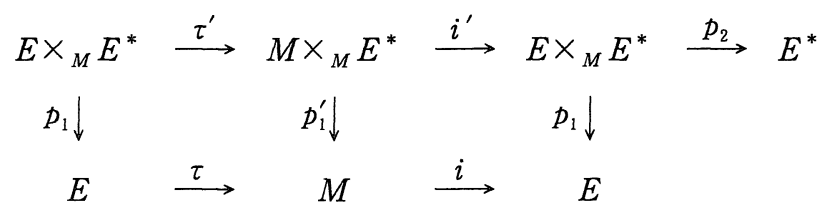

with $i$ being the embedding of $M$ as the zero section of $E$.

For morphism $\alpha$ suffice it to prove that

$$
\tau_{!} p_{1 *} \mathbf{R} \Gamma_{P^{\prime}} H \rightarrow \tau_{!} p_{1 *} H
$$

is an isomorphism for $H \in \mathrm{Ob}\left(\mathrm{D}_{\mathrm{R}^{-}}^{\mathrm{b}}\left(E \times{ }_{M} E^{*}\right)\right)$. We have a commutative diagram

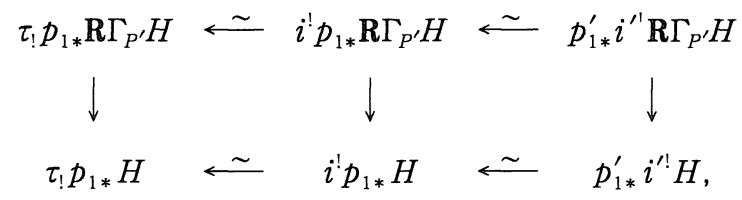

where the left square with horizontal isomorphisms is induced from morphism of functors 


$$
i^{!} \cong \tau_{!} i_{1} i^{!} \stackrel{\sim}{\longrightarrow} \tau_{!}
$$

on $\mathrm{D}_{\mathrm{R}^{+}}^{\mathrm{b}}(E)$ and the right square is the base change for the right cartesian square of (A.3). Since $i^{\prime}\left(M \times{ }_{M} E^{*}\right) \subset P^{\prime}$, the third vertical arrow is an isomorphism; therefore so is $\alpha$. We shall now prove $\beta$ to be an isomorphism. Since $p_{2}=p_{2} \circ i^{\prime} \circ \tau^{\prime}$, we have

$$
p_{1 *} p_{2} ! G^{\wedge}=p_{1 *} \tau^{\prime !} i^{\prime !} p_{2}{ }^{!} G^{\wedge} \cong \tau^{\prime} i^{!} p_{1 *} p_{2}{ }^{!} G^{\wedge}
$$

by the base change for diagram (A.3). Hence it is sufficient to remark that the morphism of functors from $\mathrm{D}^{\mathrm{b}}(M)$ to $\mathrm{D}_{\mathbf{R}^{+}}^{\mathrm{b}}(E)$

$$
\tau^{!} \rightarrow \tau^{!} \tau_{!} \tau^{!}
$$

is an isomorphism, which follows from the isomorphism $\tau_{1} \tau^{!} \cong i^{!} \tau^{!}=\mathrm{id}$ on $\mathrm{D}^{\mathrm{b}}(M)$.

Q.E.D.

As a corollary of A.1, we have :

Corollary A.2. There is a canonical distinguished triangle in $\mathrm{D}_{\mathbf{R}^{+}}^{\mathrm{b}}(E)$ :

$$
G \rightarrow \tau^{!} \tau_{!} G \rightarrow p_{1 *}^{+} p_{2}^{+!} G^{\wedge} \stackrel{+1}{\longrightarrow}
$$

where $p_{1}^{+}=\left.p_{1}\right|_{P^{+}}$and $p_{2}^{-}=\left.p_{2}\right|_{P^{+}}$, with

$$
P^{+}=\left\{(x, y) \in E \times{ }_{M} E^{*} \mid\langle x, y\rangle>0\right\}
$$

Remark. The Fourier transformation of sheaves was first introduced by Sato (cf. [SKK, Chap. 1]), and distinguished triangle (A.4) is also given in [SKK, Prop. 1.4.4] on sphere bundles, but there, since the triangle is obtained from the octahedral axiom, it is not proved that the first arrow $G \rightarrow \tau^{!} \tau_{!} G$ in (A.4) coincides with the canonical morphism induced from id: $\tau_{l} G \rightarrow \tau_{l} G$ by taking the Verdier adjoint. More recently the notion of Fourier-Sato transformations is reformulated for conic sheaves on vector bundles in [BMV] and in [KS, Sect. 3.7].

The following corollary is a refinement of Proposition 3.7.12 (3) of [KS].

Proposition A.3. Let $U$ be an open conic subset of $E, \gamma=U^{\text {oa }}$, the antipodal polar set of $U$. Suppose $\tau(U)=M$. For $G \in \mathrm{Ob}\left(\mathrm{D}_{\mathrm{R}^{+}}^{\mathrm{b}}(E)\right)$, there is a canonical commutative diagram : 
(A.5)

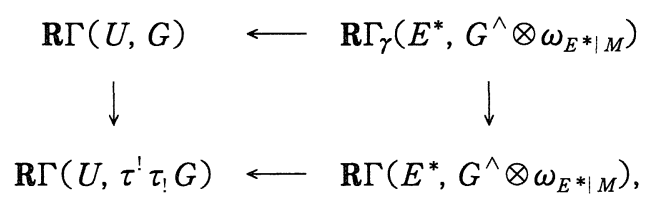

where $\omega_{E{ }^{*}{ }_{M}}=\pi^{!} k_{M}$. In this diagram, each horizontal arrow is an isomorphism if $U$ is convex in each fibre.

Proof. By Lemma A.1, we have a commutative diagram with horizontal isomorphisms :

(A.6)

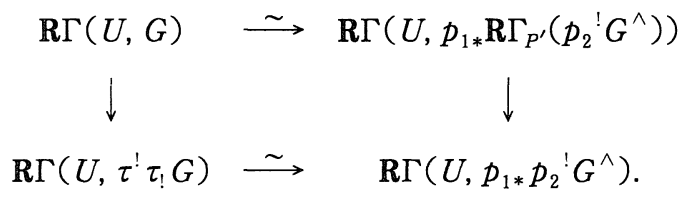

By duality, we have

$$
\begin{aligned}
\mathbf{R} \Gamma\left(U, p_{1 *} \mathbf{R} \Gamma_{P^{\prime}}\left(p_{2}{ }^{\prime} G^{\wedge}\right)\right) & \cong \mathbf{R H o m}\left(k_{U}, p_{1 *} \mathbf{R} \Gamma_{P^{\prime}}\left(p_{2}{ }^{\prime} G^{\wedge}\right)\right) \\
& \cong \mathbf{R H o m}\left(p_{2 !}\left(k_{U \times_{M} E^{*}}\right)_{P^{\prime}}, G^{\wedge}\right)
\end{aligned}
$$

and

$$
\mathbf{R} \Gamma\left(U, p_{1 *} p_{2} ! G^{\wedge}\right) \cong \mathbf{R H o m}\left(p_{2 !} k_{\left.U \times_{M} E^{*}, G^{\wedge}\right) .}\right.
$$

On the other hand we have a commutative diagram

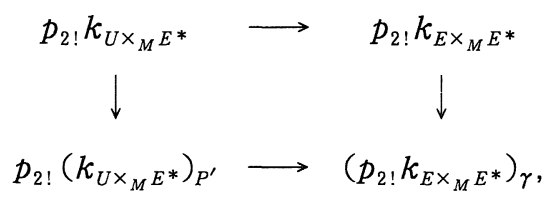

and $p_{2 !} k_{E \times_{M} E^{*}} \cong o r_{E^{*} \mid M}[-d]$. Hence we get a commutative diagram :

$$
\begin{array}{ccc}
\mathbf{R} \Gamma\left(U, p_{1 *} \mathbf{R} \Gamma_{P^{\prime}}\left(p_{2} ! G^{\wedge}\right)\right) & \longleftarrow & \mathbf{R} \Gamma_{\gamma}\left(E^{*}, G^{\wedge} \otimes \omega_{E^{*} \mid M}\right) \\
\downarrow & \downarrow \\
\mathbf{R} \Gamma\left(U, p_{1 *} p_{2} ! G^{\wedge}\right) & \longleftarrow & \mathbf{R} \Gamma\left(E^{*}, G^{\wedge} \otimes \omega_{E^{*} \mid M}\right) .
\end{array}
$$

From (A.6) and (A.8), we obtain diagram (A.5). If the fibre of $\left.\tau\right|_{U}$ is convex, by the calculation of [KS, Lemma 3.7.10], each horizontal arrow in diagram (A.7) is an isomorphism; hence the same holds in (A.8), therefore in (A.5).

Q.E.D. 


\section{Appendix B. Boundary Value Morphism and Microlocalization}

Let $X$ be a $C^{2}$-manifold, $M$ a submanifold of $X$ of codimension $d \geq 1$, $j: M \hookrightarrow X$ the embedding map, $\tau: T_{M} X \rightarrow M$ the normal bundle of $M$ in $X$,

$$
\nu_{M}: \mathrm{D}^{\mathrm{b}}(X) \rightarrow \mathrm{D}_{\mathbf{R}^{+}}^{\mathrm{b}}\left(T_{M} X\right)
$$

the specialization functor $\left[\mathrm{KS}\right.$, Sect. 4.2]. For $F \in \mathrm{Ob}\left(\mathrm{D}^{\mathrm{b}}(X)\right)$, we have the canonical morphism

$$
\nu_{M}(F) \rightarrow \tau^{!} \mathbf{R} \tau_{!} \nu_{M}(F) \cong \tau^{-1} j^{!} F \otimes \tau^{!} k_{M} .
$$

Applying the functor $H^{0}$, we have a sheaf homomorphism

$$
b: H^{0} \nu_{M}(F) \rightarrow \tau^{-1} H_{M}^{d}(F) \otimes o r_{M \mid X},
$$

with $o r_{M \mid X}$ being the relative orientation sheaf for $M \rightarrow X$.

Let $U$ be an open conic subset of $T_{M} X$. If $\left.\tau\right|_{U}: U \rightarrow M$ has connected (nonempty) fibres on $M$, (B.2) gives

$$
b_{U}: \Gamma\left(U, H^{0} \nu_{M}(F)\right) \rightarrow \Gamma\left(M, H_{M}^{d}(F) \otimes o r_{M \mid X}\right) .
$$

This is nothing but the boundary value map for $F$. Note that we have a canonical map

$$
H^{0}\left(U, \nu_{M}(F)\right) \rightarrow \Gamma\left(U, H^{0} \nu_{M}(F)\right)
$$

and an isomorphism

$$
H^{0}\left(U, \nu_{M}(F)\right) \cong \lim _{\vec{V}} H^{0}(V, F),
$$

where $V$ runs over all open subsets of $X$ satisfying $C_{M}(X \backslash V) \cap U=\varnothing[\mathrm{KS}$, Thm. 4.2.3]. Hence, from (B.3), we get a canonical map

$$
H^{0}(V, F) \rightarrow \Gamma\left(M, H_{M}^{d}(F) \otimes o r_{M \mid X}\right) .
$$

Remark. If $X$ is a complex manifold and $F=\mathcal{O}_{X}$, the formulation of boundary value morphism given here above is well known in the theory of hyperfunctions [SKK, Chap. 1].

Let $\pi: T_{M}^{*} X \rightarrow M$ be the conormal bundle of $M$ in $X$,

$$
\mu_{M}: \mathrm{D}^{\mathrm{b}}(X) \rightarrow \mathrm{D}_{\mathrm{R}^{+}}^{\mathrm{b}}\left(T_{M}^{*} X\right)
$$


the microlocalization functor [KS, Sect. 4.3].

Let $U$ be an open conic subset of $T_{M} X$, with convex (non-empty) fibres on $M$. Then we have a canonical isomorphism [KS, Prop.3.7.12]

$$
\mathbf{R} \Gamma\left(U, \nu_{M}(F)\right) \cong \mathbf{R} \Gamma_{\gamma}\left(T_{M}^{*} X, \mu_{M}(F) \otimes \omega_{T_{M}^{*} X \mid M}\right)
$$

for $F \in \mathrm{Ob}\left(\mathrm{D}^{\mathrm{b}}(X)\right)$, where $\gamma=U^{\mathrm{oa}}$ and $\omega_{T_{M}^{*} X \mid M}=\pi^{1} k_{M}\left(\cong \pi^{-1} o r_{M \mid X}[d]\right)$. Since we have a support morphism

$$
\mathbf{R} \Gamma_{\gamma}\left(T_{M}^{*} X, \mu_{M}(F) \otimes \omega_{T_{M}^{*} X \mid M}\right) \rightarrow \mathbf{R} \Gamma\left(T_{M}^{*} X, \mu_{M}(F) \otimes \omega_{T_{M}^{*} X \mid M}\right)
$$

and the target is isomorphic to

$$
\mathbf{R} \Gamma\left(M, \mathbf{R} \pi_{*} \mu_{M}(F) \otimes o r_{M \mid X}[d]\right) \cong \mathbf{R} \Gamma\left(M, \mathbf{R} \Gamma_{M} F \otimes o r_{M \mid X}[d]\right),
$$

isomorphism (B.5) is considered a boundary value morphism. This definition of boundary morphism is adopted in [ST, Sect.4]. By Proposition A.3, (B.5) is compatible with morphism (B.1); to be precise, we have :

Proposition B.1. Let $U$ be an open conic subset of $T_{M} X, \gamma=U^{\text {oa }}$. Suppose $\tau(U)=M$. There is a canonical commutative diagram:

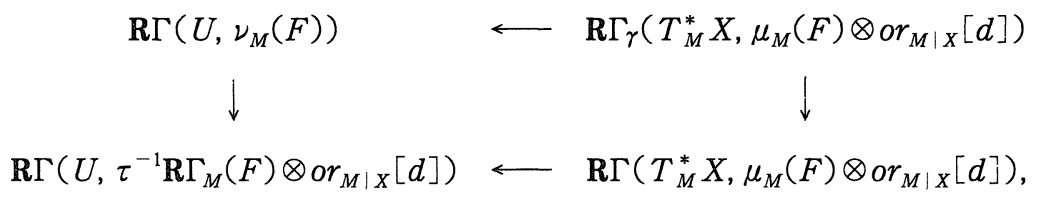

where each horizontal arrow is an isomorphism if $U$ is convex in each fibre.

For an object $F$ of $\mathrm{D}^{\mathrm{b}}(X), \mathrm{SS}(F)$ denotes its micro-support (cf. [KS, Sect.5.1]). The following is a direct consequence of isomorphism (B.5) and a geometric estimate for $\mu_{M}(F)$ [KS, Cor.5.4.10].

Proposition B.2. Let $F \in \mathrm{Ob}\left(\mathrm{D}^{\mathrm{b}}(X)\right)$. Let $U, U^{\prime}$ be open conic subsets of $T_{M} X$ with non-empty convex fibres on $M$. Suppose $U \subset U^{\prime}$. If $\operatorname{SS}(F) \cap U^{\text {oa }} \subset U^{\prime \text { oa }}$, then

$$
\mathbf{R} \Gamma\left(U^{\prime}, \nu_{M}(F)\right) \rightarrow \mathbf{R} \Gamma\left(U, \nu_{M}(F)\right)
$$

is an isomorphism.

Proof. Set $\gamma=U^{\circ a}, \gamma^{\prime}=U^{\prime o a}$. We have a commutative diagram 


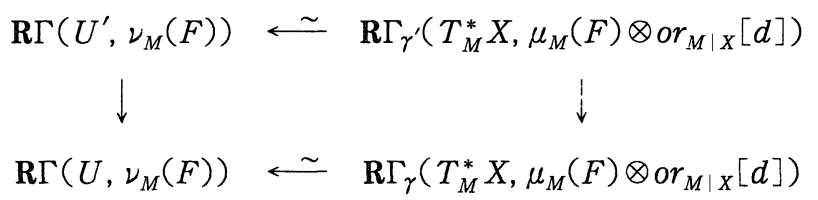

with horizontal isomorphisms. Since

$$
\operatorname{supp}\left(\mu_{M}(F)\right) \subset \operatorname{SS}(F) \cap T_{M}^{*} X
$$

[KS, Cor. 5.4.10], by the assumption, the right vertical arrow is an isomorphism.

Q.E.D.

\section{References}

[AT1] Andronikof, E. and Tose, N., Elliptic boundary value problems in the space of distributions, exp. 3, Kōkyūroku, RIMS, Kyoto Univ., 845 (1993), 13-18.

[AT2] Aoki, T. and Tajima, S., On a generalization of Bochner's tube theorem for generic CR-submanifolds, Proc. Japan Acad., Ser. A, 63 (1987), 302-303.

[BR] Baouendi, M.S. and Rothschild, L.P., Extension of holomorphic functions in generic wedges and their wave front sets, Comm. P. D. E., 13 (1988), 1441-1466.

[BRT] Baouendi, M.S., Rothschild, L.P. and Treves, F., CR structure with group action and extendability of CR functions, Invent. Math., 82 (1985), 359-396.

[BMV] Brylinski, J.-M., Malgrange, B. and Verdier, J.-L., Transformée de Fourier géométrique I, C. R. Acad. Sci., Paris, 297 (1983), 55-58.

$[\mathrm{H}]$ Hörmander, L., An Introduction to Complex Analysis in Several Variables, Van Nostrand, Princeton, 1966.

[KK] Kashiwara, M. and Kawai, T., On the boundary value problem for elliptic system of linear partial differential equations I-II, Proc. Japan Acad., Ser. A, 48 (1972), 712-715, ibid 49 (1973), 164-168.

[KS] Kashiwara, M. and Schapira, P., Sheaves on Manifolds, Springer-Verlag, 1990.

[Ko] Komatsu, H., A local version of Bochner's tube theorem, J. Fac. Sci. Univ. Tokyo, Ser. IA, 19 (1972), 201-214.

[SKK] Sato, M., Kawai, T. and Kashiwara, M., Microfunctions and pseudo-differential equations, Lecture Notes Math., Springer, 287 (1973), 265-529.

[ST] Schapira, P. and Trépreau, J.-M., Microlocal pseudoconvexity and edge-of-the-wedge theorem, Duke Math. J., 61 (1990), 105-118.

[T] Tajima, S., Analyse microlocale sur les variétés de Cauchy-Riemann et problème de prolongement des solutions holomorphes des équations aux dérivées partielles, $\mathrm{Publ}$. RIMS, Kyoto Univ., 18 (1982), 911-945. 
